\title{
Pivotal of Artificial Intelligence for Next Level Medical Practices
}

\author{
Sadique Shaikh* and Tanvir Sayyed \\ Institute of Management and Science (IMS), Maharashtra, India \\ *Corresponding author: Sadique Shaikh, Institute of Management and Science (IMS), Maharashtra, India
}

Received: 䟧 April 13, 2019

Published: 眥 April 25, 2019

\begin{abstract}
Now a Days Artificial Intelligence (A.I) Science, technology and Engineering near to peak point with spreading lot of new opportunities, facilities, and possibilities to make human life more to most comfortable and easy. Hence same also applicable in medical researches and practices zone. Below I have showcased one informative model to discuss what are the possibilities, Opportunities and openings of A.I in the medical fields.
\end{abstract}

Keywords: Deep Mind, AI, USN, SAI, UAI, loT, RFID

Abbreviations: AI: Artificial Intelligence; USN: Ubiquitous Serving Networks; IoT: Internet of Things; RFID: Radio Frequency Identifications; NLP: Natural Languages Processing; DSS: Decision Support System

\section{Short Communication}

It is really correct to say manmade AI become more advanced from God made Natural Intelligence (NI) because of high degree of sensation, accuracy and task with high speed, calibration and precise control with time management, therefore not now, but from very beginning stage A.I widely used and implemented in medical practices and healthcare related diagnosis and treatment but scope was limited [1-3]. Hence now a days due to next level advancement in A.I with high speed processors, U.A.I, S.A.I, Bionic processors, Deep Mind learning, machine learning advanced natural languages processing (NLP), Image processing, speech / smell scanning and recognition it's became most useful in medical practices and researches and acting as backbone of it as display in model like A.I based body organs implant like Artificial heart, lungs, kidney, eyes, etc. Another Useful domain is cyborgs/ cybernetics or simply "cybermatics organs" where disabled human body parts replaced with A.I based parts or sometime replacement for the reason to boost power like cyborg hand, legs etc. next one domain to focus robotics surgery and external body operation of patients with precise control and accuracy in less time [4].

Now days instead of manual or semi-automatic control fully A.I control life support system engineered for precise monitoring and save lives. Not only A.I based hardware's, but also A.I based medical software are engineered like medical diagnosis support system to examine various parameters and symptoms of patients like viral infection, root causes of diseases, blood pressure, heart beats monitoring as well as same system has inbuilt decision support system(DSS) to provide alternative treatment decisions to doctors after examine /checkups [5-7]. At several places where lack of physical doctors availability A.I based artificial doctors possible to engineer with various specialist like, nephrologist, gynecologist, cardiologist etc. which are the prototype of successful doctors with their natural intelligence (N.I) mapped and engineered with Artificial Intelligence which function exactly same like original one, hence availability of single one successful doctors skills / expertise of several places artificially . It is also possible and happened to A.I based Nano robots which insert inside the body for complicated scanning/ tracing and diagnosis where external mechanism fails to do so [8]. In last I would like to switch your attention towards the field in which you can refer my papers available online, using A.I and IoT in this concept A.I based software through IoT can perform same surgical operation around the world hospital stations with commands and controls successfully using RFIDs and USN Connected with satellite and IoT [9] (Figure 1). 


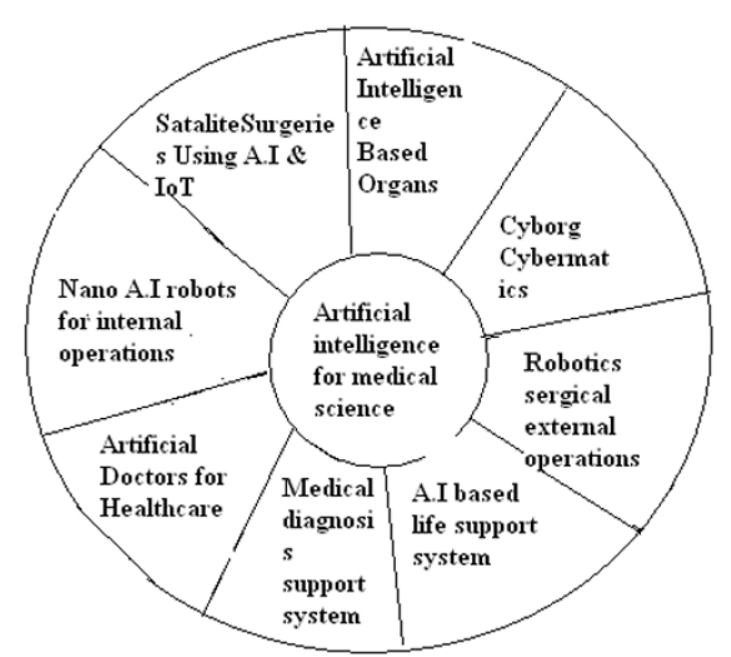

Figure 1.

\section{Acknowledgement}

I would specially acknowledge this work to my Sister Azra Sadiya who helped me while I am preparing script for typing help due to my busy schedule. I would like to credit this work to my loving wife Safeena Khan, my angels Md. Nameer Shaikh, Md. Shadaan Shaikh and my loving friend Tanveer Sayyed.

\section{References}

1. Sadique Shaikh (2013) Analysis and modeling of Strong A.I to engineer BIONIC brain for humanoid robotics application. American Journal of Embedded System and Applications 1(2): 27-36.

2. Sadique Shaikh (2017) Ultra Artificial Intelligence (UAI): Redefing AI fir New Research Dimension. in Advanced Robotics \& Automation (ARA) 6(2): 163.

3. Sadique Shaikh (2017) Fundamental Engineering for Brain-Computer Interfacing (BCI): Initiative for Neuron-Command Operating Devices. Computational Biology and Bioinformatics (CBB 5(4): 50-56.
4. Sadique Shaikh (2018) Defining ultra-artificial intelligence (UAI) implementation using bionic (biological-like-electronics) brain engineering insight. MOJ App Bio Biomech 2(2): 127-128.

5. Sadique Shaikh (2018) Insight Artificial to Cyborg Intelligence Modeling. Arch Ind Engg 1(1): 1- 4.

6. Sadique Shaikh (2018) Artificial Intelligence Engineering for Cyborg Technology Implementation in Robotics \& Automation Engineering Journal. Robot Autom Eng J 3(1).

7. Sadique Shaikh (2018) Engineering Insight for Humanoid Robotics Emotions and Violence with Reference to System Error 1378. Robot Autom Eng J 3(2).

8. Sadique Shaikh (2018) Defining Cyborg Intelligence for Medical and Super-Human Domains. Trends in Technical \& Scientific Research 2(3).

9. Sadique Shaikh (2018) Ultra artificial intelligence (UAI) Engineering for robotics violence control, detect and corrective measures. International Robotics \& Automation Journal 4(4).

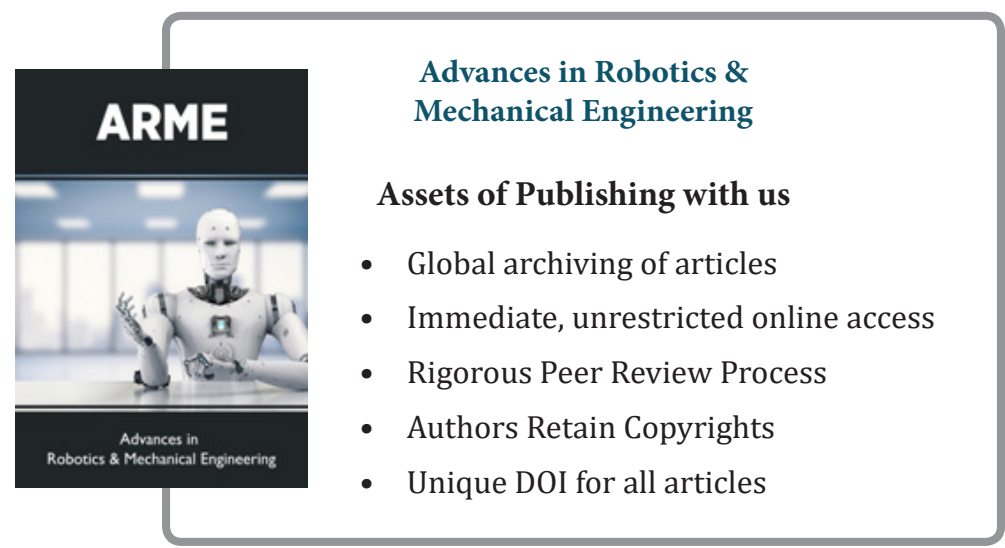

